\title{
Using GIS and Kriging to Analyze the Spatial Distributions of the Health Risk of Indoor Air Pollution
}

\author{
Kevin Fong-Rey Liu1 ${ }^{*}$, Ming-Jui Hung1, Jong-Yih Kuo², Han-Hsi Liang ${ }^{3}$ \\ ${ }^{1}$ Department of Safety, Health and Environmental Engineering, Ming Chi University of Technology, New Taipei \\ City, Taiwan \\ ${ }^{2}$ Department of Science and Information Engineering, National Taipei University of Technology, Taipei, Taiwan \\ ${ }^{3}$ Department of Architecture, National United University, Miaoli, Taiwan \\ Email: ${ }^{*}$ kevinliu@mail.mcut.edu.tw
}

Received 27 April 2015; accepted 19 August 2015; published 25 August 2015

\begin{abstract}
Kriging is an interpolation technique that is used to estimate a variable at an unmeasured location from observed values at nearer locations. In this study, it is used to analyze the spatial distributions of the health risk of indoor air pollution. The study case is an air-conditioned office building that has 16 floors, located in Taipei, Taiwan. The Kriging method is used in drawing health risk maps on the basis of limited sample points and facilitates investigating the possible source of pollution.
\end{abstract}

\section{Keywords}

Kriging, Health Risk Assessment, Hazard Index, Air-Conditioned Office Building

\section{Introduction}

Clean air is a basic requirement for human life. In particular, the indoor air quality of homes, offices, schools, health care centers, and private and public buildings is critical for human health because most people cannot be separated from these spaces. However, some buildings, building materials, and interior equipment or even cooking and heating fuel combustion may release harmful substances that cause serious health damage. Furthermore, people spend approximately $80 \%$ - 90\% of their time inside office buildings or other structures; poor indoor air quality can cause sick-building syndrome, directly affecting work quality and efficiency and negatively affecting health [1].

Indoor air pollutants that are defined in the Taiwan Indoor Air Quality Act include carbon dioxide $\left(\mathrm{CO}_{2}\right)$, carbon monoxide (CO), formaldehyde (HCHO), total volatile organic compounds (TVOCs), bacteria, fungi, airborne particles with a particle diameter $\leq 10 \mu \mathrm{m}\left(\mathrm{PM}_{10}\right)$, airborne particles with a diameter $\leq 2.5 \mu \mathrm{m}\left(\mathrm{PM}_{2.5}\right)$, and ozone $\left(\mathrm{O}_{3}\right)$. $\mathrm{CO}_{2}$ can cause choking at high concentrations (more than $\left.7000 \mathrm{ppm}\right)$. CO combines with he-

${ }^{*}$ Corresponding author.

How to cite this paper: Liu, K.F.-R., Hung, M.-J., Kuo, J.-Y. and Liang, H.-H. (2015) Using GIS and Kriging to Analyze the Spatial Distributions of the Health Risk of Indoor Air Pollution. Journal of Geoscience and Environment Protection, 3, 20-25.

http://dx.doi.org/10.4236/gep.2015.36004 
moglobin in the blood to reduce the oxygen supply to the body, causing headaches, decreased alertness, flu-like symptoms, nausea, fatigue, rapid breathing, chest pain, confusion, and impaired judgment. HCHO, as a carcinogen, can also cause a dry or sore throat, nosebleeds, headaches, fatigue, memory and concentration problems, nausea, dizziness, breathlessness, and a burning, stinging pain in the eyes. Exposure to TVOCs has carcinogenic and noncarcinogenic effects. The noncarcinogenic effects include damage to the liver or kidney systems and respiratory irritation or discomfort. Gram-negative bacteria infection usually causes fever, weakness, pain, and shock, which can develop into more serious illnesses such as typhoid fever, urinary tract infection, and meningitis. Gram-positive bacteria affect the structure or function of specific cells and cause diseases such as gas gangrene, tetanus, botulism, diphtheria, and scarlet fever. Toxic fungi can cause human allergies and infection. Common allergic reactions are allergic asthma and allergic rhinitis. Repeated exposure to high concentrations of a fungus can cause hypersensitivity pneumonitis. Fungal infection often occurs on the skin and mucosal surfaces. In addition to infection and allergy, parts of fungal metabolites can be toxic, with some containing mycotoxins. Suspended particulates in the alveoli and trachea result in allergic rhinitis, asthma, chronic obstructive pulmonary disease, and other diseases. Indoor $\mathrm{O}_{3}$ causes a change in lung function, which results in respiratory problems.

The study case is an analysis of health risk in an office building in Taipei. At any sampling point, it is easy to ascertain whether the measured value exceeds the regulatory standards and subsequently to calculate its health hazards. However, at nonsampled points, the possible concentrations and corresponding health effects caused by indoor air pollutants can only be interpolated by using spatial statistical methods such as Kriging, which has been applied in environmental studies [2] [3]. This study uses Kriging as an optimal interpolation method, which is based on a regression against observed $z$ values of surrounding data points that are weighted according to spatial covariance values.

\section{Materials and Methods}

\subsection{Case Study}

A 16-floor office building located in Taipei, Taiwan, has 70 sample points in the building, with approximately four to five measurement points per floor. The 7th, 8th, and 12th floors are illustrated in this paper. The distributions of their measurement points are shown in Figures 1-3, respectively.

\subsection{Health Risk Assessment}

Health risk assessment (HRA) is a process for estimating the characteristics and probability of adverse health effects occurring in humans who may be exposed to hazardous substances. Its four basic ingredients are hazard

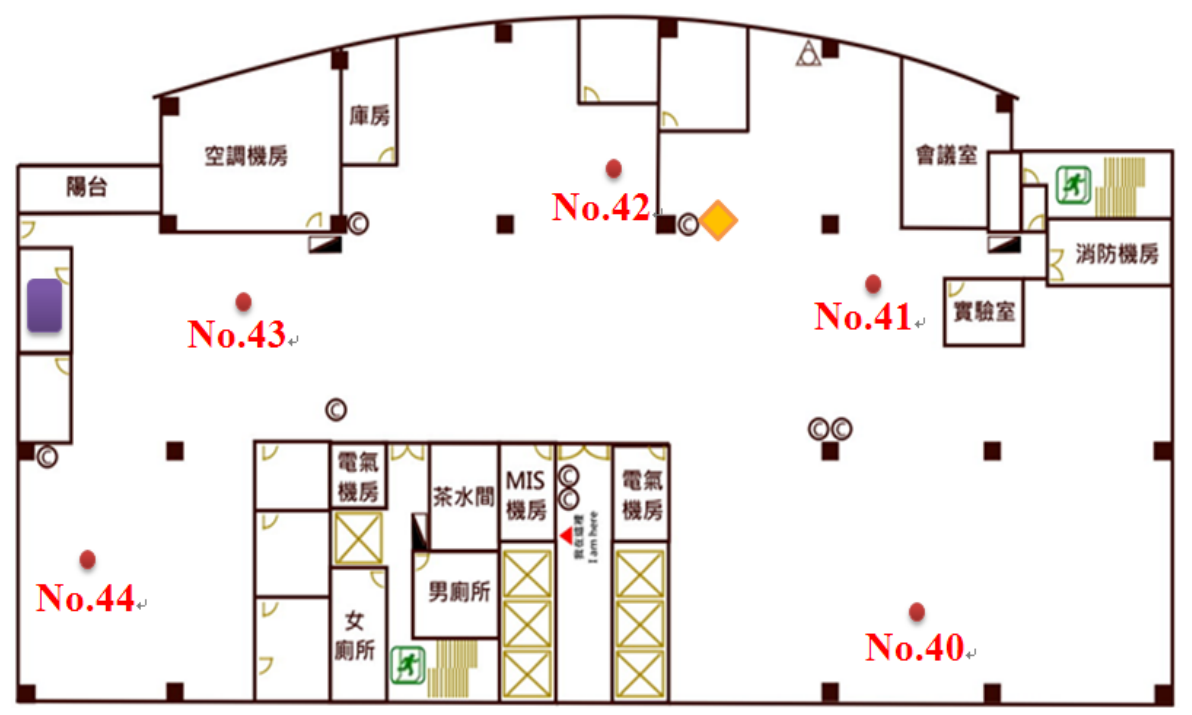

Figure 1. Distribution of measurement points over the 7th floor. 


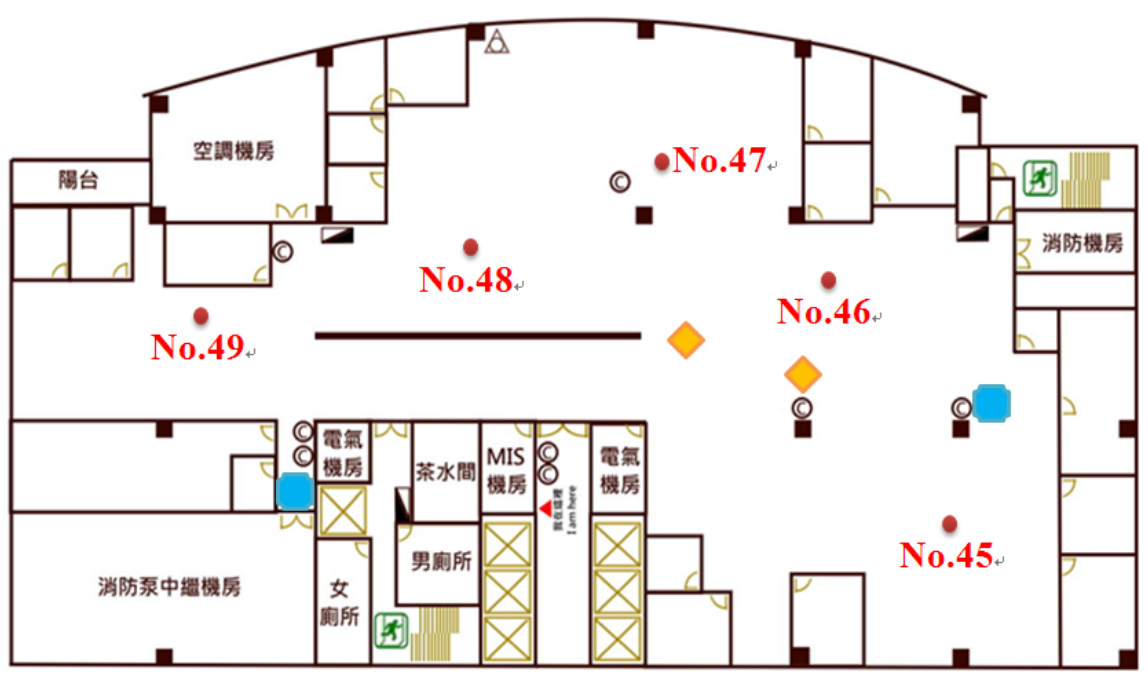

Figure 2. Distribution of measurement points over the 8th floor.

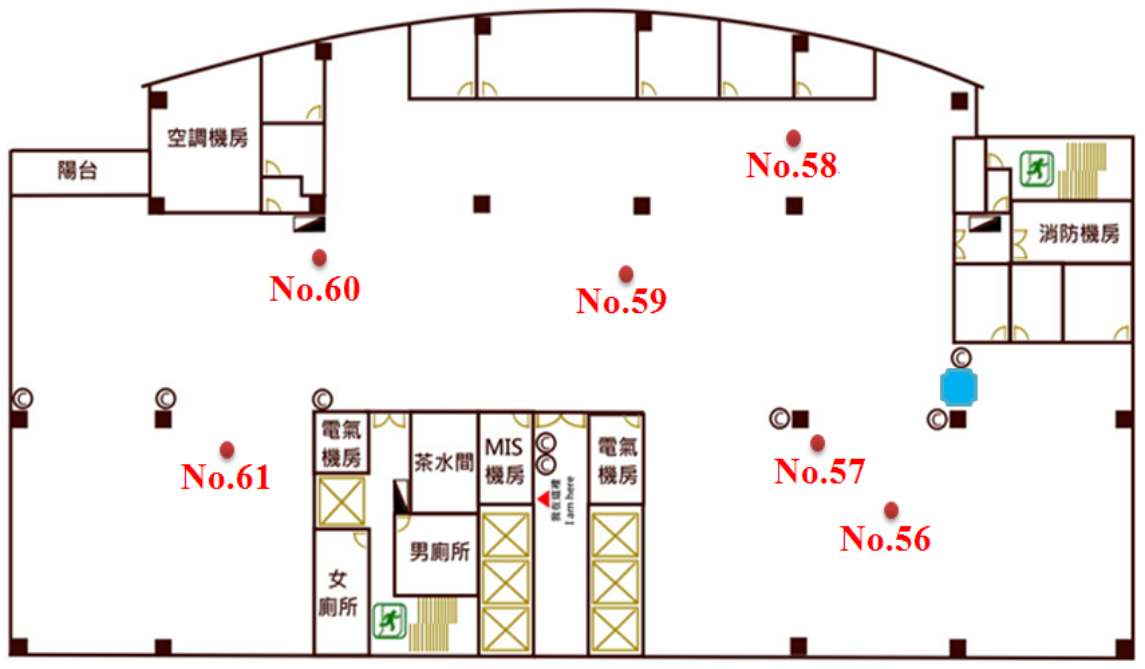

Figure 3. Distribution of measurement points over the 12th floor.

identification, exposure assessment, dose-response assessment, and risk characterization [4]. Hazard identification aims to recognize any potential health problem that a substance can cause; exposure assessment determines the amount, duration, and pattern of exposure to the substance; dose-response assessment estimates the amount of the substance required to cause varying degrees of adverse health effects; and risk characterization interprets the risk of the substance causing cancer or other illnesses. In the risk characterization of noncarcinogenic substances, the HRA of a substance can be evaluated by using its hazard quotient (HQ), the ratio of the intake of a hazardous substance to its reference dose. Multiple hazardous substances may affect the same organ (or organ system) causing a joint effect; hence, a target organ-specific hazard index is used to sum the HQ scores of multiple substances that have a joint effect on a specific organ [5].

Noncancer risk is usually determined by comparing the actual level of chemical exposure to the level of exposure that is not expected to cause any adverse effects, even in the most susceptible people [6]. The level of concern for noncarcinogenic contaminants is determined by calculating an HQ or hazard index (HI). An HI is the sum of the HQs for several chemicals that affect the same target organ. If the HQ or HI equals or exceeds one, there may be exposure to site contaminants.

$$
\mathrm{HQ}=\frac{C}{R f C}
$$


where $C$ indicates the exposure concentration $\left(\mu \mathrm{g} / \mathrm{m}^{3}\right)$, and $R f C$ indicates the reference concentration $\left(\mu \mathrm{g} / \mathrm{m}^{3}\right)$.

$$
\mathrm{HI}=\sum_{i} \mathrm{HQ}_{i}=\sum_{i} \frac{C_{i}}{R f C_{i}}
$$

where $C_{i}$ indicates the exposure concentration of the $i^{\text {th }}$ substance $\left(\mu \mathrm{g} / \mathrm{m}^{3}\right)$, and $R f C_{i}$ indicates the reference concentration of the $i^{\text {th }}$ substance $\left(\mu \mathrm{g} / \mathrm{m}^{3}\right)$.

\subsection{Geographic Information System and Kriging}

A geographic information system (GIS) is a computer system for capturing, storing, checking, and displaying data related to positions on the Earth's surface that can show many data types on one map, enabling patterns and relationships to be easily observed, analyzed, and understood. Regression methods and geostatistics are some of the analytical tools that are usually available in a GIS. Kriging is a group of geostatistical techniques to interpolate the value of a random field at an unobserved location from observations of its value at nearby locations. Kriging is an interpolation technique in which the surrounding measured values are weighted to derive a predicted value for an unmeasured location. Weights are based on the distance between the measured points, prediction locations, and overall spatial arrangement among the measured points. Kriging is unique among interpolation methods in which it provides an easy method for characterizing the variance, or precision, of predictions. Kriging is based on regionalized variable theory, which assumes that the spatial variation in the data being modeled is homogeneous across a surface. In other words, the same pattern of variation can be observed at all surface locations.

\section{Results}

The hazard indices of indoor air pollution over measurement points $40,41,42,43$, and 44 for the 7 th floor were 1.33, 1.14, 0.95, 1.13, and 0.91, respectively; their interpolated map of health risk is shown in Figure 4.

The hazard indices of indoor air pollution over measurement points $45,46,47,48$, and 49 for the 8th floor were 1.27, 0.92, 0.87, 0.96, and 0.99, respectively; their interpolated map of health risk is shown in Figure 5.

The hazard indices of indoor air pollution over measurement points 56, 57, 58, 59, 60, and 61 for the 12th floor were 1.23, 1.14, $0.95,1.09,1.15$, and 1.05, respectively; their interpolated map of health risk is shown in Figure 6.

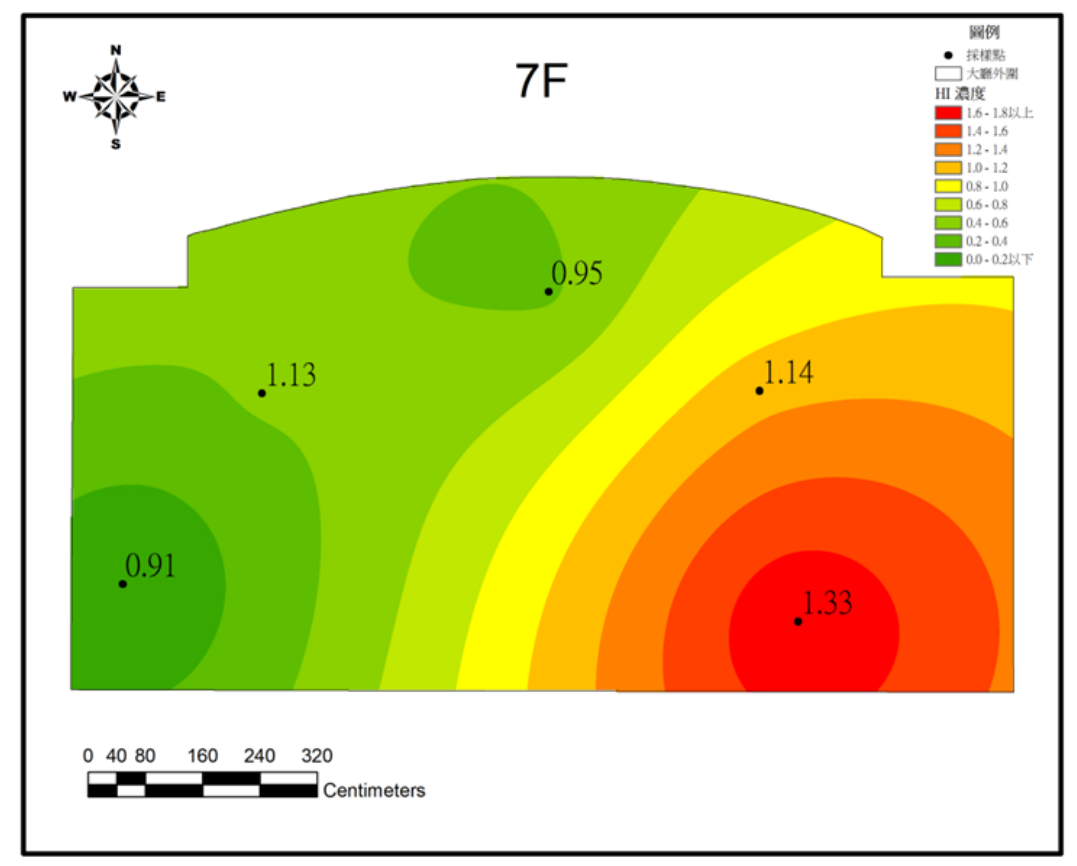

Figure 4. Interpolated map of health risk for the 7th floor. 


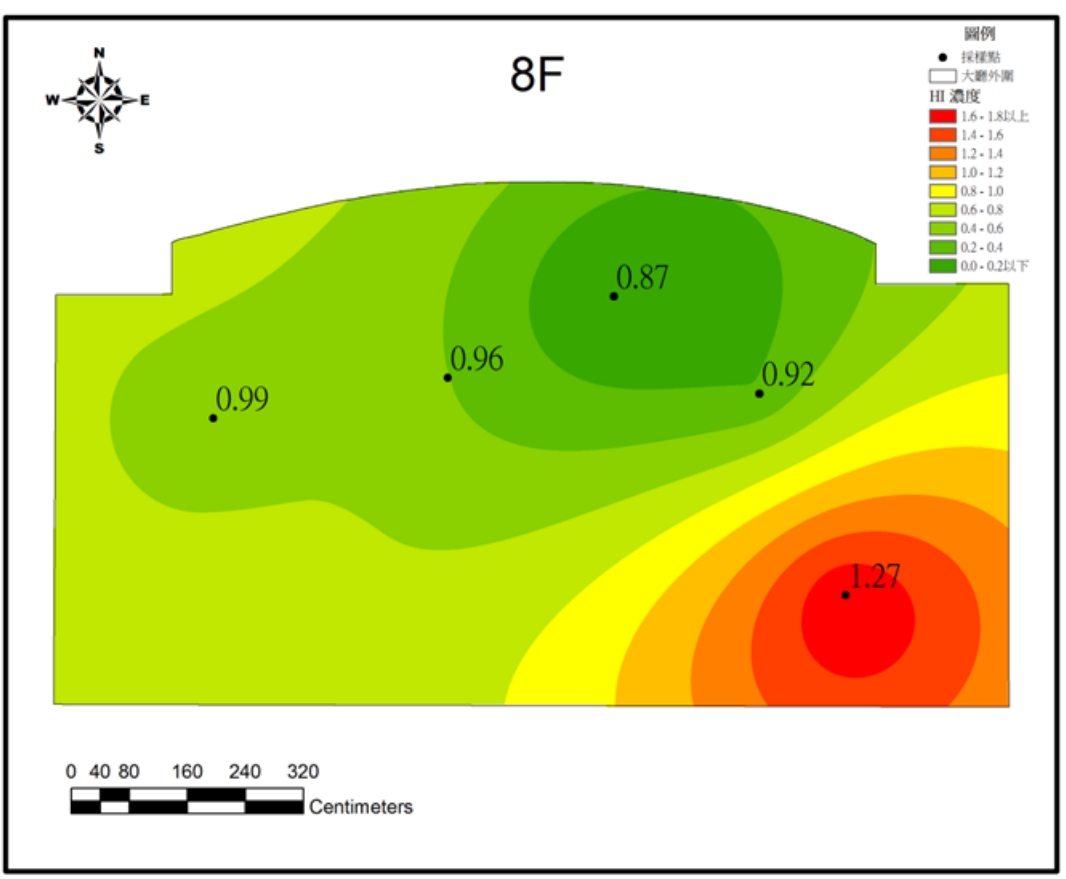

Figure 5. Interpolated map of health risk for the 8th floor.

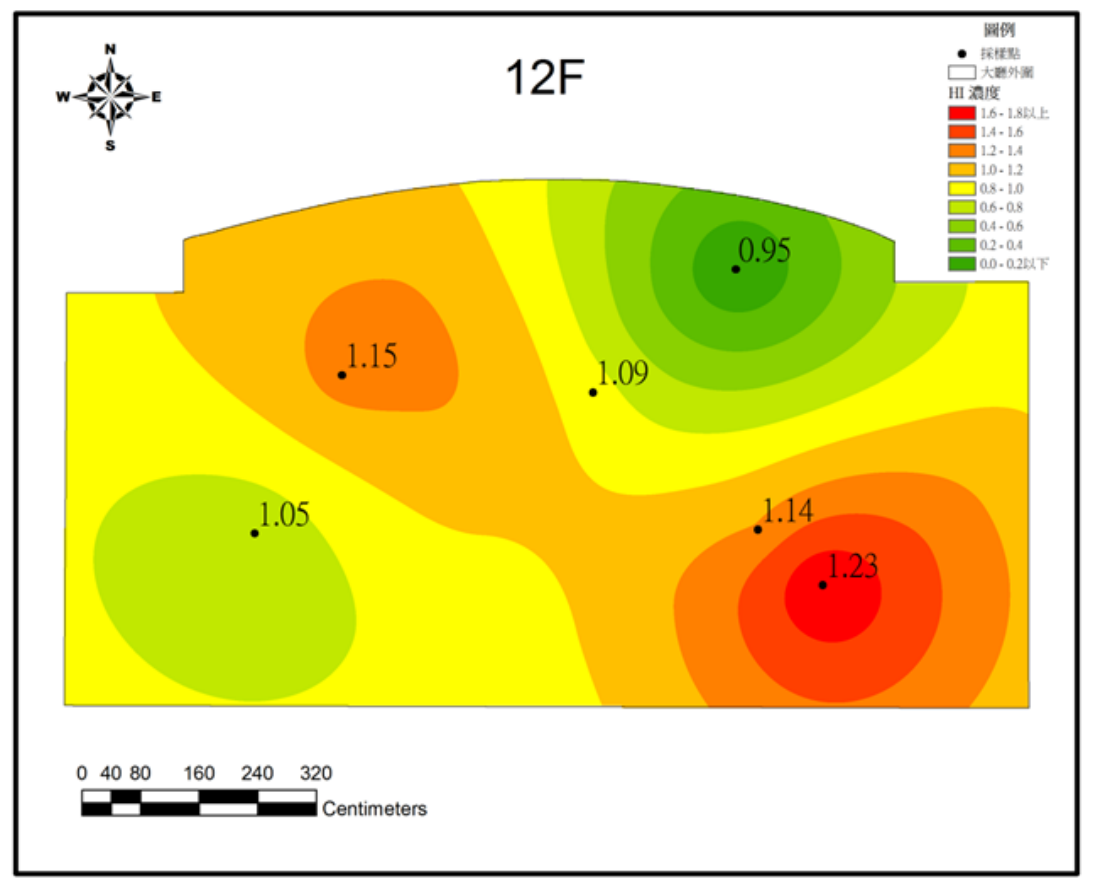

Figure 6. Interpolated map of health risk for the 12th floor.

\section{Discussion and Conclusion}

The risk analysis shows a sample point (1.43\% of 70 sample points) with possible harm to the cardiovascular system, two sample points (2.86\% of 70 sample points) with possible harm to the eyes, and 24 sample points (34.29\% of 70 sample points) with possible harm to the respiratory system. Therefore, those involved in the study case should pay particular attention to respiratory harm. The study recommends the following measures to improve indoor air quality: First, identify the sources of pollution, move them to a separate space, and install a 
separate exhaust system in the space; second, adjust the ventilation system; third, during renovation or reconstruction, enhance the ventilation rate to increase the amount of fresh air; fourth, close doors and windows temporarily and shut down the ventilation function of the central air-conditioning system in instances of high concentrations of outdoor air pollution.

\section{Acknowledgements}

The authors thank the Ministry of Science and Technology of Taiwan for financially supporting this research under Contract MOST 103-2221-E-131-001-MY2.

\section{References}

[1] Wolkoff, P. (2010) Indoor Air Pollutants in Office Environments: Assessment of Comfort, Health, and Performance. International Journal of Hygiene and Environmental Health, 216, 371-394. http://dx.doi.org/10.1016/j.ijheh.2012.08.001

[2] Vu, V.H., Le, X.Q., Pham, N.H. and Hens, L. (2013) Application of GIS and Modelling in Health Risk Assessment for Urban Road Mobility. Environmental Science Pollution Research, 20, 5138-5149. http://dx.doi.org/10.1007/s11356-013-1492-5

[3] Tyagi, A. and Singh, P. (2013) Applying Kriging Approach on Pollution Data Using GIS Software. International Journal of Environmental Engineering and Management, 4, 185-190.

[4] WHO (2011) WHO Human Health Risk Assessment Toolkit: Chemical Hazards. ISBN: 924154807X.

[5] U.S. EPA (2013) National-Scale Air Toxics Assessment for 1996: Draft for EPA Science Advisory Board Review. BiblioGov, ISBN: 1289217556.

[6] California EPA (2001) A Guide to Health Risk Assessment. 